\title{
Safety, efficacy, and analysis of key parameters after prophylactic administration of a sustained-release formulation of azithromycin in lung cancer surgery
}

\author{
Daisuke Taniguchi $^{1,2 \#}$, Hironosuke Watanabe ${ }^{1 \#}$, Yoshitomo Morinaga ${ }^{3,4}$, Daisuke Sasaki ${ }^{4}$, \\ Junichi Matsuda ${ }^{4}$, Shuntaro Sato ${ }^{5}$, Norihito Kaku ${ }^{4}$, Takuro Miyazaki ${ }^{1}$, Keitaro Matsumoto ${ }^{1,2}$, \\ Tomoshi Tsuchiya ${ }^{1}$, Toshiyuki Sakaeda ${ }^{6}$, Katsunori Yanagihara ${ }^{4}$, Takeshi Nagayasu ${ }^{1,2}$
}

${ }^{1}$ Department of Surgical Oncology, Nagasaki University Graduate School of Medicine, Nagasaki, Japan; ${ }^{2}$ Medical-Engineering Hybrid Professional Development Program, Nagasaki University Graduate School of Biomedical Sciences, Nagasaki, Japan; ${ }^{3}$ Department of Microbiology, Graduate School of Medicine and Pharmaceutical Sciences, University of Toyama, Toyama, Japan; ${ }^{4}$ Department of Laboratory Medicine, Nagasaki University, Nagasaki, Japan; ${ }^{5}$ Nagasaki University Hospital Clinical Research Center, Nagasaki, Japan; ${ }^{6}$ Department of Pharmacokinetics, Kyoto Pharmaceutical University, Kyoto, Japan

Contributions: (I) Conception and design: H Watanabe, Y Morinaga, T Tsuchiya; (II) Administrative support: T Miyazaki, K Matsumoto, T Nagayasu; (III) Provision of study materials or patients: N Kaku, K Yanagihara; (IV) Collection and assembly of data: D Taniguchi, D Sasaki, J Matsuda, T Sakaeda; (V) Data analysis and interpretation: D Taniguchi, S Sato; (VI) Manuscript writing: All authors; (VII) Final approval of manuscript: All authors.

\#These authors contributed equally to this work.

Correspondence to: Takeshi Nagayasu. Department of Surgical Oncology, Nagasaki University Graduate School of Biomedical Sciences, 1-7-1 Sakamoto, Nagasaki 852-8501, Japan. Email: nagayasu@nagasaki-u.ac.jp.

Backgroundk The current use of prophylactic antibiotics for lung cancer surgery requires modification in aging individuals with impaired lung function. A sustained-release formulation of azithromycin (AZM-SR) could help resolve some of these challenges with its sustained antibacterial and anti-inflammatory effects. The aim of this study was to examine the safety and efficacy of AZM-SR in lung cancer surgery as well as its anti-inflammatory effect.

Methods: Fifty patients were included in the study, and AZM-SR was administered 1 day prior to the surgery. The clinical course, including postoperative complications, was monitored, and the concentration of AZM, bacterial culture, and inflammatory cytokine levels of resected lung specimens were evaluated.

Results: No side effects related to AZM-SR were observed. Five cases of postoperative pneumonia (10\%) were observed; technical issues were involved in 3 cases. All patients recovered well. Four cases showed positive bacterial culture upon lung tissue examination; however, this was not significantly correlated with postoperative complications. A negative correlation was observed between AZM concentration in lung tissue and interleukin-6 (IL-6) expression.

Conclusions: Prophylactic utilization of AZM-SR in lung cancer surgery seems feasible. The anti-inflammatory effect of AZM might contribute additional beneficial effects in the perioperative management of lung cancer surgery.

Keywords: Azithromycin (AZM); lung cancer surgery; prophylactic

Submitted Feb 14, 2021. Accepted for publication Mar 23, 2021.

doi: 10.21037/apm-21-383

View this article at: http://dx.doi.org/10.21037/apm-21-383

$\wedge$ ORCID: 0000-0003-1702-9021. 


\section{Introduction}

The number of elderly patients, along with various preoperative complications, has been increasing worldwide (1-4). As lung cancer patients often have other complications such as respiratory diseases (e.g., chronic obstructive pulmonary disease) probably caused by smoking, such underlying diseases, besides lung cancer, could affect both the incidence of postoperative complications as well as long-term prognosis $(1-3,5,6)$. The prophylactic use of antibiotics has traditionally included cephem antibiotics for a few days in the field of general thoracic surgery $(7,8)$. However, Since the number of older patients with chronic lung disease or impaired lung function has increased, cephem antibiotics might not be sufficient for preventing postoperative infectious complications, especially postoperative pneumonia (8). Therefore, novel strategies are urgently required in the use of prophylactic antibiotics.

Azithromycin (AZM) is a macrolide antibiotic that targets gram-positive bacteria as well as gram-negative rods and mycoplasma (9-11). The effects of this drug last for 7 days after administration. AZM has been indicated for respiratory tract and skin infections (9-11).

Recently, a single-administration type, sustained-release formulation of AZM, Azithromycin-2 g SR (AZM-SR) has been found to accumulate at high concentrations in the target organs, show broad spectrum for respiratory infection, and ensure simple and secure compliance by single administration (12). Moreover, AZM modulates immune reactions by suppressing inflammatory cytokine release (13-17). Recently, AZM has been reported to reduce lung inflammation and $M U C 5 B$ gene expression, which is responsible for encoding a major core protein secreted from the respiratory tract that is correlated with inflammation in the respiratory system $(13,15)$.

Considering the above findings, we hypothesized that AZM-SR could be an ideal drug formulation for prophylactic administration in general thoracic surgery. Hence, the purpose of this study was to examine the efficacy of single-dose AZM in general thoracic surgery. The risk factors related to postoperative pneumonia were investigated. Moreover, we determined the levels of AZM in the resected lung specimens and performed bacterial culture of the resected lung specimens. In addition, the efficacy of AZM in modulating inflammatory cytokines in clinical patients was investigated. We present the following article in accordance with the STROBE reporting checklist (available at http://dx.doi.org/10.21037/apm-21-383).

\section{Methods}

\section{Patient background and study design}

This trial was a non-blinded clinical pilot study. The patients who would receive surgery for lung cancer were enrolled in this study between 2013 and 2015. Under informed consent, Azithromycin-2 g SR (AZM-SR) was administered after the evening meal, 1 day prior to the surgery. Regular antibiotic prophylaxis was not administered. The primary endpoint was any adverse event caused by AZM-SR, which would help in assessing the safety of AZM-SR during the perioperative period. The secondary endpoints were the incidence of postoperative infection including postoperative pneumonia, the need for re-administration of antibiotics, and postoperative mortality at 30 days post-surgery.

Exclusion criteria included patients with allergy to macrolides, QT prolongation syndrome, severe renal dysfunction, fructose intolerance, severe liver dysfunction (Child-Pugh C), and current treatment with other antibiotics.

Postoperative pneumonia was defined by the presence of a new or progressive infiltrate on chest radiography or computed tomography during the patient's hospitalization, together with any of the following: new-onset purulent sputum, change in the character of chronic sputum, fever $\geq 38^{\circ} \mathrm{C}$, a new rise in C-reactive protein (CRP) value or white blood cell count, positive blood cultures or isolation of the pathogen from sputum, and transtracheal aspirate (18) (Figure 1).

\section{Ethical approval and informed consent}

The study was conducted in accordance with the Declaration of Helsinki (as revised in 2013). This study was approved by the institutional review board at Nagasaki University Hospital (Approval number; 12121779-7) and was registered in the University Medical Information Network (UMIN) Center (Clinical trial number; UMIN000010368). After the detailed explanation of studyrelated information and documents, only the patients from whom written informed consent was obtained were enrolled in this study.

\section{Bacterial culture}

Bacteria were cultured from lung specimens that were 


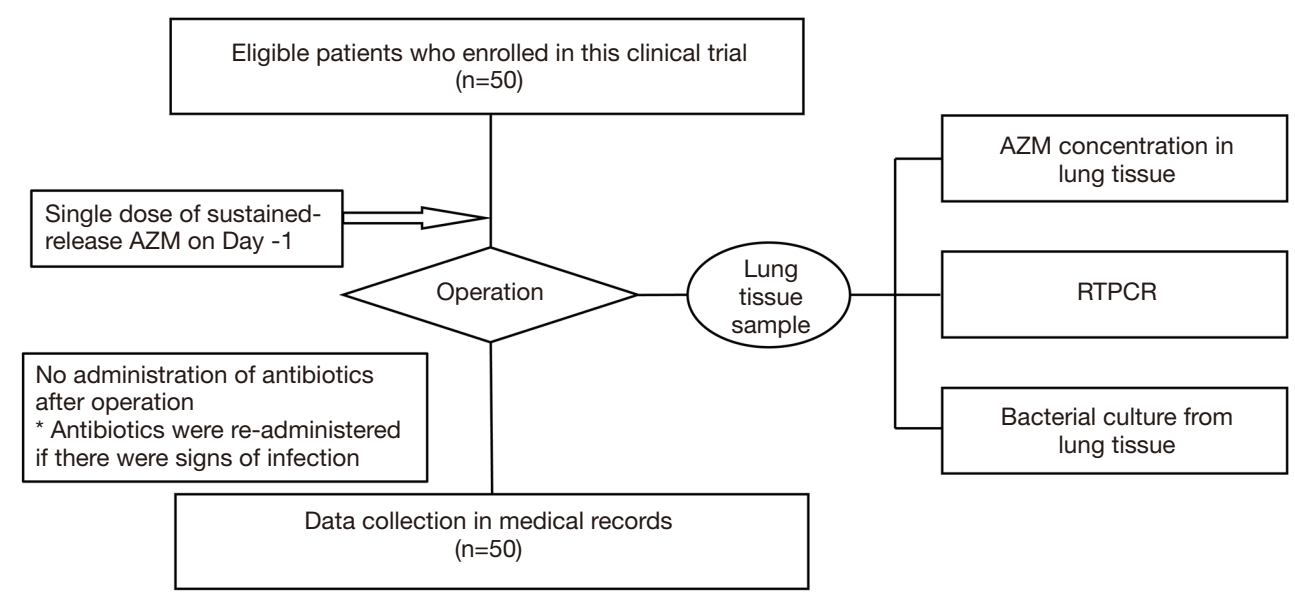

Figure 1 Scheme of the study protocol. Eligible patients who agreed to participate in this clinical trial by written informed consent were enrolled in this study. All participants took the single dose of sustained-release AZM on the night before the operation. Lung tissue samples without any tumor lesion were obtained during surgery. After the operation, no additional antibiotics were administered. *Antibiotics were re-administered only to patients who showed signs of infection. The postoperative course was observed carefully, and clinical data were collected in the medical records. RT-PCR, real time-polymerase chain reaction; AZM, azithromycin.

excised under sterile conditions. Each lung specimen was collected and stored in an anaerobic culture bottle for culture on HK semisolid medium. If bacterial growth was confirmed, further cultures were performed using chocolate/blood agar medium, bromothymol blue lactate/ chromo agar medium, and Colombia agar medium. Bacterial strain detection was performed by genomic sequencing if $16 \mathrm{~S}-\mathrm{rRNA}$ amplification was detected among culture-positive cases.

\section{AZM concentration in excised lung tissue}

The evaluation of AZM concentrations in the excised lung was performed by using the liquid chromatography/tandem mass spectrometry (LC/MS/MS) protocol as previously described (19). A lung specimen, $1 \mathrm{~cm}^{2}$ in size, was obtained from excised lung tissue (not the tumor lesion) at the time of surgery and was stored at $-80{ }^{\circ} \mathrm{C}$. The weighed lung sample was homogenized, and the concentration of AZM was determined by LC/MS/MS as previously described (19).

\section{RNA extraction and quantitative PCR}

RNA extraction from resected lung specimens and qPCR were performed as previously described (20). Briefly, the excised lung tissue sample, $1 \mathrm{~cm}$ in size, was stored in RNAlater ${ }^{\circledR}$, and total RNA was extracted by using a
PureLink RNA micro kit (Life Technologies, CA, USA). One microgram of total RNA was reverse-transcribed into cDNA using a SuperScript VILO cDNA synthesis kit (Life Technologies, Carlsbad, CA, USA).

The expression of $M U C 5 B$, tumor necrosis factoralpha (TNF- $\alpha$ ), and IL- 6 was measured using a cDNA template with LightCycler 480 probes Master Mix (Roche Diagnostics) and a LightCycler480 PCR System (Roche Diagnostics, Mannheim, Germany). To normalize the results for variability in concentration and integrity of RNA and cDNA, the GAPDH gene was used as an internal control for each sample. PCR primers and TaqMan probes are listed in the Supplemental Table.

\section{Statistical analysis}

Data are reported as mean \pm standard deviation (SD). All statistical analyses were performed using JMP Pro software (version 14.2.0; SAS Institute, Cary, NC, USA). Comparisons between groups were performed using the Wilcoxon signed-rank test and $t$-test, and nominal scale analysis involved Pearson $\chi^{2}$. We also used Spearman rank correlation to further investigate the relationship between AZM concentration in the lung tissue and other variables. A total of 20 hypotheses were tested, and Bonferroni correction was used for counteracting the multiple testing problem. Values of $\mathrm{P}<0.0025$ were considered as 
Table 1 Patient baseline demographics and characteristics for the overall population

\begin{tabular}{|c|c|}
\hline Parameter & Value $(n=50)$ \\
\hline Age (year) & $70 \pm 9.0$ \\
\hline Sex (male/female) & $25 / 25$ \\
\hline $\mathrm{BMI}\left(\mathrm{kg} / \mathrm{m}^{2}\right)$ & $22.7 \pm 2.9$ \\
\hline Smoker & 27 \\
\hline Pack-year & $15 \pm 31.7$ \\
\hline \multicolumn{2}{|l|}{ Comorbidity/history } \\
\hline Hypertension & 12 \\
\hline Previous malignancies & 11 \\
\hline Respiratory disease & 10 \\
\hline Heart disease & 6 \\
\hline Thyroid disease & 5 \\
\hline Diabetes & 2 \\
\hline \multicolumn{2}{|c|}{ Preoperative blood examination } \\
\hline CRP (mg/dL) & $0.065 \pm 0.4$ \\
\hline $\mathrm{TP}(\mathrm{g} / \mathrm{dL})$ & $7.1 \pm 0.47$ \\
\hline ALB (g/dL) & $4.2 \pm 0.33$ \\
\hline Cre (mg/dL) & $0.76 \pm 0.21$ \\
\hline BUN (mg/dL) & $16 \pm 4.32$ \\
\hline eGFR $\left(\mathrm{mL} / \mathrm{min} / 1.73 \mathrm{~m}^{2}\right)$ & $69.3 \pm 15.3$ \\
\hline AST (IU/L) & $21 \pm 6.9$ \\
\hline ALT (IU/L) & $16 \pm 8.9$ \\
\hline ALP (IU/L) & $243 \pm 59.4$ \\
\hline T-Bil (mg/dL) & $0.7 \pm 0.33$ \\
\hline LDH (IU/L) & $186 \pm 29.7$ \\
\hline \multicolumn{2}{|l|}{ Operation } \\
\hline Lobectomy & 42 \\
\hline Segmentectomy & 7 \\
\hline Partial resection & 1 \\
\hline Complete VATS & 33 \\
\hline Operation time (min) & $226.5 \pm 59.0$ \\
\hline Bleeding amount (g) & $100 \pm 195.9$ \\
\hline \multicolumn{2}{|l|}{ Stage } \\
\hline 0 & 3 \\
\hline I & 41 \\
\hline II & 1 \\
\hline III & 4 \\
\hline NA & 1 \\
\hline
\end{tabular}

Table 1 (continued)
Table 1 (continued)

\begin{tabular}{lc}
\hline Parameter & Value $(\mathrm{n}=50)$ \\
\hline Pathology & 42 \\
Adenocarcinoma & 3 \\
Squamous cell carcinoma & 2 \\
LCNEC & 1 \\
Adenosquamous cell carcinoma & 2 \\
Others & \\
\hline
\end{tabular}

Data are reported as mean \pm standard deviation (SD). NA, not applicable; CRP, C-reactive protein; TP, total protein; ALB, albumin; Cre, creatinine; BUN, blood urea nitrogen; eGFR, estimated glomerular filtration rate; AST, aspartate aminotransferase; ALT, alanine aminotransferase; ALP, alkaline phosphatase; T-Bil, total bilirubin; $\mathrm{LDH}$, lactate dehydrogenase; VATS, video-assisted thoracoscopic surgery; LCNEC, large cell neuroendocrine carcinoma.

significant.

\section{Results}

\section{Patient characteristics}

Fifty patients were enrolled in this study and received a single dose of AZM-SR on the day before the operation. The patients' age was $70 \pm 9.0$ years [mean \pm standard deviation (SD)], and the body mass index was $22.7 \pm 2.9 \mathrm{~kg} / \mathrm{m}^{2}$. Twenty-five (50\%) patients were male, and 27 (54\%) were ex- or current smokers with an average pack-years of smoking of 15 (range, $0-104)$. The comorbidity and past history were as follows: 12 cases of hypertension, 11 cases of previous malignancy, 10 cases of respiratory diseases, and 5 cases of thyroid diseases. All of the average data of the parameters of preoperative blood examination were within the normal range. The surgical procedure consisted of lobectomy in 42 cases, segmentectomy in 7 cases, and wedge resection in 1 case. Thirty-three cases received the operation under complete video-assisted thoracoscopic surgery conditions. Operation time and bleeding amount during surgery were $226.5 \pm 59.0 \mathrm{~min}$ and $100 \pm 195.9 \mathrm{~g}$, respectively. Pathological stage I and adenocarcinoma were the most common types of lung cancer (41 cases and 42 cases, respectively) (Table 1 ).

\section{Clinical postoperative outcome}

Postoperative pneumonia was observed in 5 cases (10\%), 3 
Table 2 Postoperative outcome and data

\begin{tabular}{lc}
\hline Parameter & Value $(\mathrm{n}=50)$ \\
\hline Maximum of postoperative CRP $(\mathrm{mg} / \mathrm{dL})$ & $8.0 \pm 5.98$ \\
Postoperative pneumonitis & $5(10 \%)$ \\
Arrhythmia & $3(6 \%)$ \\
Chylothorax & $2(4 \%)$ \\
Prolonged air leakage & $1(2 \%)$ \\
Late-onset bronchopleural fistula & $1(2 \%)$ \\
Urinary tract infection & $1(2 \%)$ \\
Re-administration of antibiotics & $9(18 \%)$ \\
AZM-related postoperative adverse event & $0(0 \%)$ \\
Postoperative mortality & $0(0 \%)$ \\
AZM concentration in the lung ( $\mu \mathrm{g} / \mathrm{g})$ & $5.9 \pm 5.9$ \\
Bacterial detection in lung tissue culture & $4(8 \%)$ \\
Relative mRNA level & \\
IL6/GAPDH & $0.37 \pm 0.52$ \\
MUC5B/GAPDH & $0.00034 \pm 0.018$ \\
TNF- $\alpha / G A P D H$ & $0.0044 \pm 0.15$ \\
\hline
\end{tabular}

Data are reported as mean \pm standard deviation (SD). CRP, C-reactive protein; AZM, azithromycin; TP, total protein; IL6, interleukin-6; TNF- $\alpha$, tumor necrosis factor-alpha; GAPDH, gyceraldehyde-3-phosphate dehydrogenase.

of which had technical issues including bronchial stenosis. In two cases, a slight bronchial stenosis, due to bronchial kinking following lung resection, was observed during radiological examination. The remaining one showed a strong adhesin between the lung and chest wall, which required extensive dissection. When abnormal shadow was found in the remaining lungs following the postoperative period in these three cases, it was difficult to distinguish pneumonia from atelectasis or lung hematoma. However, we included these three cases as pneumonia according to our definition of postoperative pneumonia. All cases were treated by re-administration of antibiotics and recovered. Arrhythmia (atrial fibrillation, paroxysmal supraventricular tachycardia) and chylothorax were observed as postoperative complications; these cases were treated with conservative treatment and recovered well. Re-administration of antibiotics was conducted in nine cases (19\%), including lung hematoma, chylothorax, and bronchial stenosis. In this study, antibiotics were re-administered under the consultation of an infectious disease physician; Tazobactam/
Piperacillin was the most commonly used antibiotics (5 cases). The peak value of postoperative $\mathrm{C}$-reactive protein (CRP) was $8.0 \pm 5.98 \mathrm{mg} / \mathrm{dL}$. The AZM concentration in lung tissue was $5.9 \pm 5.9 \mu \mathrm{g} / \mathrm{g}$. There was no adverse event related to AZM administration, and postoperative mortality was $0 \%$ (Table 2).

\section{Bacterial culture of lung tissue}

Five patients showed bacterial growth from the resected lung specimens, which did not show any significant correlation with postoperative pneumonia (Table 3).

\section{Inflammatory cytokine analysis}

The relative gene expression of interleukin-6 (IL-6), tumor necrosis factor-alpha $(T N F-\alpha)$, and $M U C 5 B$ normalized by glyceraldehyde-3-phosphate dehydrogenase (GAPDH) expression in the resected specimens was $0.37 \pm 0.52$, $0.00034 \pm 0.018$, and $0.0044 \pm 0.15$, respectively.

\section{Correlation analysis for lung AZM concentrations}

Correlations between AZM concentrations in lung tissue and clinical/laboratory findings were assessed statistically. As a result, we observed a negative correlation between AZM concentration and relative IL-6 mRNA levels (IL-6/GAPDH); no other significant correlation was detected (Figure 2).

\section{Analysis for postoperative pneumonia, re-administration of antibiotics, and bacterial detection in lung tissue culture}

Correlation analysis was performed between clinical/ laboratory data and postoperative pneumonia $(n=5)$, readministration of antibiotics ( $n=9)$, and bacterial detection by lung tissue culture ( 4 positive cases). In the analysis for postoperative pneumonia, significant differences were observed in heart disease as a comorbidity; however, no other significant correlation was observed including AZM concentrations in lung tissue and the relative mRNA levels obtained by polymerase chain reaction (PCR) analysis. No significant correlation was observed in the analysis of readministration of antibiotics and tissue culture (Table 3).

\section{Discussion}

In this study, we have focused on the prophylactic use of AZM-SR in general thoracic surgery and illustrated the 
Table 3 Univariate analysis of the results in patients who experienced postoperative pneumonitis, patients who needed re-administration of antibiotics, and those who tested positive for bacterial lung tissue culture

\begin{tabular}{|c|c|c|c|}
\hline Parameter & $\begin{array}{l}\text { Postoperative pneumonitis } \\
\qquad(\mathrm{n}=5)\end{array}$ & $\begin{array}{l}\text { Re-administration of } \\
\text { antibiotics }(n=9)\end{array}$ & $\begin{array}{l}\text { Bacteria detection by lung tissue } \\
\text { cultivation (positive case }=4 \text { ) }\end{array}$ \\
\hline Age (year) & 0.1123 & 0.5025 & 0.2823 \\
\hline Sex (male/female) & 0.6374 & 0.7128 & 1 \\
\hline Weight (kg) & 0.9484 & 0.3247 & 0.9572 \\
\hline \multicolumn{4}{|l|}{ Comorbidity/history } \\
\hline Respiratory disease $(n=10)$ & 0.2386 & 0.854 & 0.2971 \\
\hline Heart disease $(n=6)$ & 0.0005 & 0.0296 & 0.4413 \\
\hline eGFR $\left(\mathrm{mL} / \mathrm{min} / 1.73 \mathrm{~m}^{2}\right)$ & 0.0433 & 0.7332 & 0.9857 \\
\hline AST (IU/L) & 0.2237 & 0.0226 & 0.1318 \\
\hline ALT (IU/L) & 0.056 & 0.0103 & 0.0958 \\
\hline Complete VATS ( $n=33)$ & 0.1958 & 0.4651 & 0.4813 \\
\hline Operation time (min) & 0.0485 & 0.161 & 0.7611 \\
\hline Bleeding amount (g) & 0.0244 & 0.0687 & 0.3612 \\
\hline Maximum postoperative CRP level (mg/dL) & 0.0076 & 0.0083 & 0.0535 \\
\hline Bacterial detection by lung tissue culture & 0.487 & 0.3286 & \\
\hline
\end{tabular}

Data are reported as mean \pm standard deviation (SD). Cre, creatinine; BUN, blood urea nitrogen; eGFR, estimated glomerular filtration rate; AST, aspartate aminotransferase; ALT, alanine aminotransferase; VATS, video-assisted thoracoscopic surgery; CRP, C-reactive protein; AZM, azithromycin; IL-6, interleukin-6; TNF- $\alpha$, tumor necrosis factor-alpha; GAPDH, gyceraldehyde-3-phosphate dehydrogenase.

feasibility of this novel concept. Moreover, we evaluated the levels of multiple cytokines associated with inflammation and mucosal immunity, which have reinforced this concept.

IL-6 mRNA levels and AZM concentrations showed negative correlation, demonstrating the anti-inflammatory effect of AZM in general thoracic surgery, while that of TNF- $\alpha$ and MUC 5B showed no correlation. The immunomodulatory effect of AZM has been investigated in lung disease patients $(14,21)$. In the acute phase of immunomodulation, AZM decreases the production of proinflammatory cytokines such as IL- 6 , TNF- $\alpha$, interleukin- 8 (IL-8), granulocyte-macrophage colony-stimulating factor (GM-CSF), and matrix metalloproteinases (MMPs) (22-25). Our data demonstrate a negative correlation between AZM concentrations in lung tissue and IL-6 mRNA levels, which is consistent with previous reports. Moreover, especially in lung transplant patients, AZM has been used for its anti-inflammatory effect (26). Our finding of the negative 
A

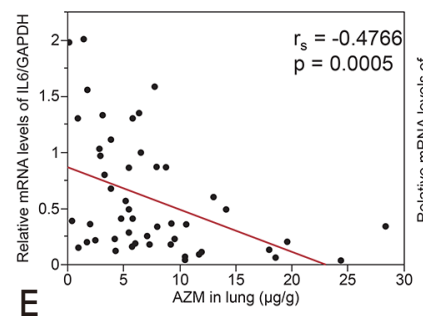

$\mathrm{E}$

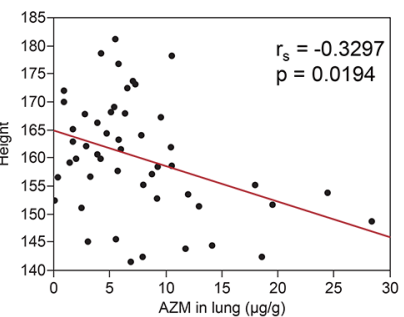

I

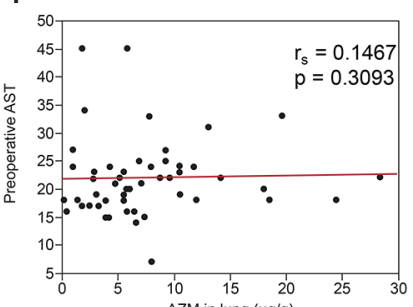

M

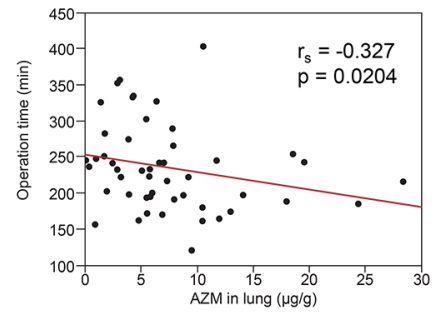

B

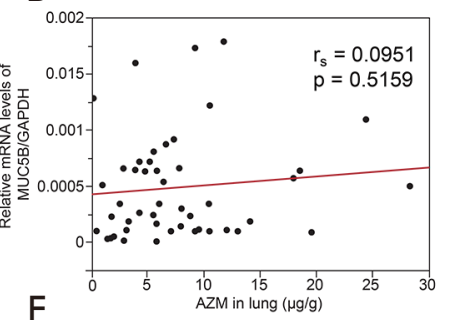

$\mathrm{F}$

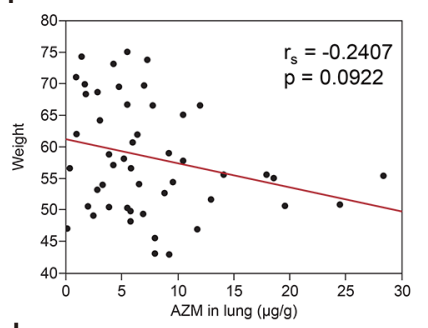

J

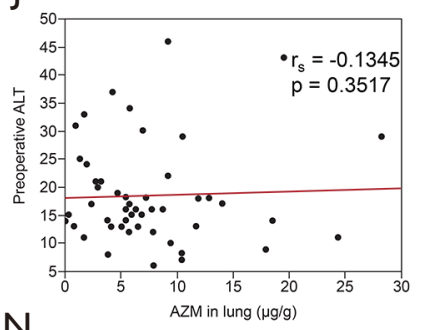

$\mathrm{N}$

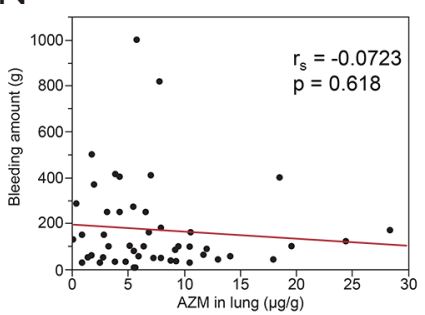

C

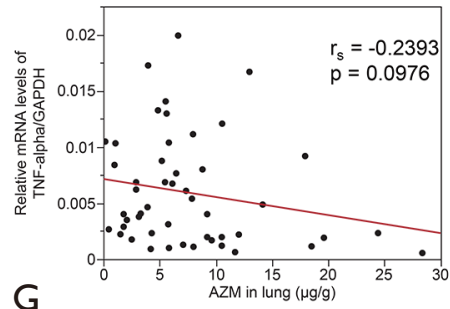

G

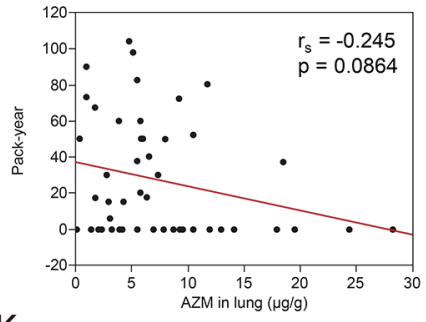

$\mathrm{K}$

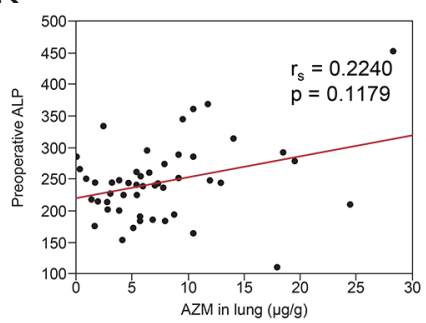

D

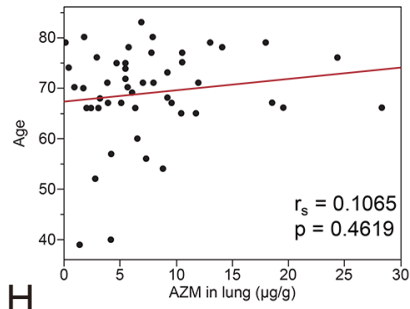

$\mathrm{H}$

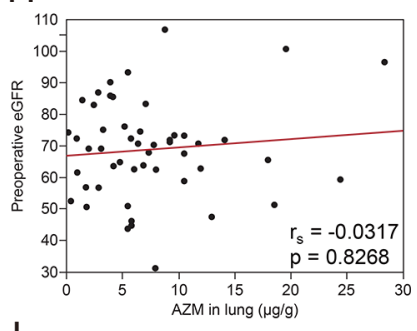

L

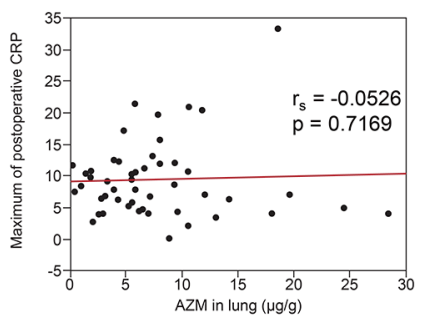

Figure 2 Correlation between the AZM concentration in the lung tissue and clinical/laboratory findings. Analysis of the correlation between the AZM concentration in the lung tissue and variables. Relative mRNA expression, (A) IL6/GAPDH, (B) MUC5B/GAPDH, (C) TNF- $\alpha /$ GAPDH; (D) age; (E) height of the patients; (F) weight of the patients; (G) pack-year index; (H) preoperative eGFR; (I) preoperative AST; (J) preoperative ALT; $(\mathrm{K})$ preoperative ALP; (L) maximum postoperative CRP value; (M) operation time; $(\mathrm{N})$ bleeding amount. AZM, azithromycin; IL-6, interleukin-6; TNF- $\alpha$, tumor necrosis factor-alpha; GAPDH, glyceraldehyde-3-phosphate dehydrogenase; eGFR, estimated glomerular filtration rate; AST, aspartate aminotransferase; ALT, alanine aminotransferase; ALP, alkaline phosphatase; CRP, C-reactive protein.

correlation between AZM and IL-6 concentrations could help inform future use of AZM in general thoracic surgery, especially for high-risk patients with impaired respiratory function. Although other cytokines showed no correlation in this study, further investigations may show correlation between AZM and other cytokines in the respiratory tract.

We found no AZM-related side effects in our study, such as elevation of liver enzymes, long QT syndrome, or diarrhea, which demonstrates the safety of AZM-SR in general thoracic surgery and suggests the feasibility of another study with increased number of patients with a double-arm study design.

The results of the bacterial culture of the resected lung specimens showed a few positive cases, which showed no correlation with postoperative infection. Thus, we concluded that AZM-SR showed no negative effect with 
respect to postoperative infection. The positive bacterial cultures of the resected lung might be due to preoperative colonization, as postoperative bacterial examination showed no positive culture. The effect of AZM-SR in the prevention of postoperative infection should be further investigated. AZM levels in pulmonary tissue have been found to remain high for up to 5 days even after a single oral $500 \mathrm{mg}$ dose, in contrast to plasma AZM levels (27). Higher AZM levels have also been reported to be higher in tissue interstitial fluid than in plasma $(19,28)$. These findings suggest that the higher AZM concentration in the respiratory tract would possess an inhibitory effect on many sensitive respiratory pathogens (27).

Postoperative pneumonia is one of the most common complications in patients with lung cancer undergoing therapeutic surgical resection and has been reported as a poor prognostic factor $(29,30)$. The reported incidence of postoperative pneumonia following lung cancer surgery ranges from $3.6 \%$ to $18.7 \%$ (29-32). The incidence of postoperative pneumonia in our study was $10 \%$ (5 cases), while that with traditional cephem antibiotics in our institution was $6.7 \%$ to $9.4 \%$ between 2013 and 2015 (data not shown). However, some technical issues were involved in 3 cases, suggesting that this AZM formulation was not inferior to classical prophylactic treatment. A significant relationship was only observed between postoperative pneumonia and heart disease as a comorbidity, which was consistent with previous reports that showed underlying heart disease as a risk factor for postoperative pneumonia $(31,33)$. Although direct correlation between $A Z M$ and postoperative pneumonia was not found, the high concentration of AZM in the lung may prevent postoperative pneumonia through an anti-inflammatory effect, as evidenced by the low $I L-6$ expression in this study. Some patients received re-administration of other antibiotics as prophylactics for lung hematoma or chylothorax; however, the sustained effect of AZM-SR observed in this study suggests that re-administration would be unnecessary.

The use of macrolides has drawn attention to possible cardiovascular events, particularly arrhythmias, mainly with concomitant use of other QT-prolonging drugs or underlying structural heart disease. However, azithromycin may be considered the safest of all macrolides in this respect as serum levels in healthy volunteers are $>3,000$ times lower than drug concentrations required for cardiomyocyte potassium channel (hERG/IKr)-blockade, action potential prolongation, and QTc prolongation (26). In this study, no
AZM-related adverse events, including typical arrhythmia (long QT), were observed. We believe that our exclusion criteria, namely, severe liver dysfunction and long QT syndrome, were appropriate in this study.

This study is not without limitations. First, since we focused on the biological features rather than the statistical aspects in this pilot study, the number of patients (sample size) was not statistically calculated, and no control group was included. Second, we did not show a direct relationship between AZM and postoperative pneumonia. However, we believe that this study demonstrates the safety and efficacy of the prophylactic use of AZM-SR in general thoracic surgery.

In conclusion, prophylactic use of AZM-SR in general thoracic surgery seems safe and feasible. Additional benefits from the anti-inflammatory effect of AZM could be expected with such use. This study lays the foundation for future studies on AZM-SR in matched patient populations and offers a new strategy of prophylactic treatment in general thoracic surgery.

\section{Acknowledgments}

Funding: None.

\section{Footnote}

Reporting Checklist: The authors have completed the STROBE reporting checklist. Available at http://dx.doi. org/10.21037/apm-21-383

Data Sharing Statement: Available at http://dx.doi. org/10.21037/apm-21-383

Conflicts of Interest: All authors have completed the ICMJE uniform disclosure form (available at http://dx.doi. org/10.21037/apm-21-383). The authors have no conflicts of interest to declare.

Ethical Statement: The authors are accountable for all aspects of the work in ensuring that questions related to the accuracy or integrity of any part of the work are appropriately investigated and resolved. The study was conducted in accordance with the Declaration of Helsinki (as revised in 2013). The study was approved by institutional review board of Nagasaki University Hospital (approval No. 12121779-7) and was registered in the University Medical Information Network (UMIN) Center (Clinical trial No. 
UMIN000010368). Informed consent for this retrospective analysis was taken from all individual participants.

Open Access Statement: This is an Open Access article distributed in accordance with the Creative Commons Attribution-NonCommercial-NoDerivs 4.0 International License (CC BY-NC-ND 4.0), which permits the noncommercial replication and distribution of the article with the strict proviso that no changes or edits are made and the original work is properly cited (including links to both the formal publication through the relevant DOI and the license). See: https://creativecommons.org/licenses/by-nc-nd/4.0/.

\section{References}

1. Wei S, Chen F, Liu R, et al. Outcomes of lobectomy on pulmonary function for early stage non-small cell lung cancer (NSCLC) patients with chronic obstructive pulmonary disease (COPD). Thorac Cancer 2020;11:1784-9.

2. Sekine Y, Kesler KA, Behnia M, et al. COPD may increase the incidence of refractory supraventricular arrhythmias following pulmonary resection for non-small cell lung cancer. Chest 2001;120:1783-90.

3. Arca JA, Lamelas IP, Ortega RA, et al. Lung cancer and COPD: a common combination. Arch Bronconeumol 2009;45:502-7.

4. Miyazaki T, Yamazaki T, Nakamura D, et al. Surgery or stereotactic body radiotherapy for elderly stage I lung cancer? A propensity score matching analysis. Surg Today 2017;47:1476-83.

5. Rochester CL, Holland AE. Pulmonary rehabilitation and improved survival for patients with COPD. JAMA 2020;323:1783-85.

6. Hodson R. Chronic obstructive pulmonary disease. Nature 2020;581:S1.

7. Oxman DA, Issa NC, Marty FM, et al. Postoperative antibacterial prophylaxis for the prevention of infectious complications associated with tube thoracostomy in patients undergoing elective general thoracic surgery: a double-blind, placebo-controlled, randomized trial. JAMA Surg 2013;148:440-6.

8. Deguchi H, Tomoyasu $M$, Shigeeda $W$, et al. Influence of prophylactic antibiotic duration on postoperative pneumonia following pulmonary lobectomy for non-small cell lung cancer. J Thorac Dis 2019;11:1155-64.

9. Bakheit AHH, Al-Hadiya BMH, Abd-Elgalil AA. Azithromycin. Profiles Drug Subst Excip Relat Methodol 2014;39:1-40.
10. Dunn CJ, Barradell LB. Azithromycin. A review of its pharmacological properties and use as 3-day therapy in respiratory tract infections. Drugs 1996;51:483-505.

11. Albert RK, Connett J, Bailey WC, et al. Azithromycin for prevention of exacerbations of COPD. N Engl J Med 2011;365:689-98.

12. Soda M, Ito S, Matsumaru N, et al. Evaluation of the microbiological efficacy of a single 2-gram dose of extended-release azithromycin by population pharmacokinetics and simulation in Japanese patients with gonococcal urethritis. Antimicrob Agents Chemother 2017;62:e01409-17.

13. Morinaga Y, Yanagihara K, Miyashita N, et al. Azithromycin, clarithromycin and telithromycin inhibit MUC5AC induction by Chlamydophila pneumoniae in airway epithelial cells. Pulm Pharmacol Ther 2009;22:580-6.

14. Yamada K, Yanagihara K, Kaku N, et al. Azithromycin attenuates lung inflammation in a mouse model of ventilator-associated pneumonia by multidrug-resistant Acinetobacter baumannii. Antimicrob Agents Chemother 2013;57:3883-8.

15. Yamada K, Morinaga Y, Yanagihara K, et al. Azithromycin inhibits MUC5AC induction via multidrug-resistant Acinetobacter baumannii in human airway epithelial cells. Pulm Pharmacol Ther 2014;28:165-70.

16. Yamashita Y, Nagaoka K, Kimura H, et al. Efficacy of azithromycin in a mouse pneumonia model against hospitalacquired methicillin-resistant Staphylococcus aureus. Antimicrob Agents Chemother 2019;63:e00149-19.

17. Liu J, Zhong X, He Z, et al. Erythromycin suppresses the cigarette smoke extract-exposed dendritic cell-mediated polarization of CD4 + T cells into Th17 cells. J Immunol Res 2020;2020:1387952.

18. Lee JY, Jin SM, Lee CH, et al. Risk factors of postoperative pneumonia after lung cancer surgery. J Korean Med Sci 2011;26:979-84.

19. Kobuchi S, Aoki M, Inoue C, et al. Transport of azithromycin into extravascular space in rats. Antimicrob Agents Chemother 2016;60:6823-7.

20. Tsushima Y, Uno N, Sasaki D, et al. Quantitative RT-PCR evaluation of a rapid influenza antigen test for efficient diagnosis of influenza virus infection. J Virol Methods 2015;212:76-9.

21. Cramer CL, Patterson A, Alchakaki A, et al. Immunomodulatory indications of azithromycin in respiratory disease: a concise review for the clinician. Postgrad Med 2017;129:493-9. 
22. Murphy DM, Forrest IA, Corris PA, et al. Azithromycin attenuates effects of lipopolysaccharide on lung allograft bronchial epithelial cells. J Heart Lung Transplant 2008;27:1210-6.

23. Gielen V, Johnston SL, Edwards MR. Azithromycin induces anti-viral responses in bronchial epithelial cells. Eur Respir J 2010;36:646-54.

24. Parnham MJ, Haber VE, Giamarellos-Bourboulis EJ, et al. Azithromycin: mechanisms of action and their relevance for clinical applications. Pharmacol Ther 2014;143:225-45.

25. Poachanukoon O, Koontongkaew S, Monthanapisut P, et al. Macrolides attenuate phorbol ester-induced tumor necrosis factor alpha and mucin production from human airway epithelial cells. Pharmacology 2014;93:92-9.

26. Ruttens D, Verleden SE, Vandermeulen E, et al. Prophylactic azithromycin therapy after lung transplantation: post hoc analysis of a randomized controlled trial. Am J Transplant 2016;16:254-61.

27. Morris DL, De Souza A, Jones JA, et al. High and prolonged pulmonary tissue concentrations of azithromycin following a single oral dose. Eur J Clin Microbiol Infect Dis 1991;10:859-61.

28. Kobuchi S, Kabata T, Maeda K, et al. Pharmacokinetics

Cite this article as: Taniguchi $\mathrm{D}$, Watanabe $\mathrm{H}$, Morinaga Y, Sasaki D, Matsuda J, Sato S, Kaku N, Miyazaki T, Matsumoto K, Tsuchiya T, Sakaeda T, Yanagihara K, Nagayasu T. Safety, efficacy, and analysis of key parameters after prophylactic administration of a sustained-release formulation of azithromycin in lung cancer surgery. Ann Palliat Med 2021;10(5):5098-5107. doi: 10.21037/apm-21-383 of macrolide antibiotics and transport into the interstitial fluid: comparison among erythromycin, clarithromycin, and azithromycin. Antibiotics (Basel) 2020;9:199.

29. Simonsen DF, Søgaard M, Bozi I, et al. Risk factors for postoperative pneumonia after lung cancer surgery and impact of pneumonia on survival. Respir Med 2015;109:1340-6.

30. Schussler O, Alifano M, Dermine H, et al. Postoperative pneumonia after major lung resection. Am J Respir Crit Care Med 2006;173:1161-9.

31. Yang R, Du C, Xu J, et al. Excessive intravenous crystalloid infusion after video-assisted thoracoscopic surgery lobectomy is associated with postoperative pneumonia. J Cardiothorac Surg 2019;14:209.

32. Arslantas MK, Kara HV, Tuncer BB, et al. Effect of the amount of intraoperative fluid administration on postoperative pulmonary complications following anatomic lung resections. J Thorac Cardiovasc Surg 2015;149:314-20.

33. Pavia R, Spinelli F, Monaco M, et al. Lung cancer and cardiovascular diseases: occurrence, comorbidity and surgical timing. J Cardiovasc Surg (Torino) 2007;48:227-31. 
Supplementary

\begin{tabular}{|c|c|c|}
\hline Gene name (Accession no.) & & Primer and probe sequence \\
\hline \multirow{2}{*}{ IL-6 (NM_000600) } & $\mathrm{R}$ & 5'- TGGCATTTGTGGTTGGGTCAG-3' \\
\hline & $\mathrm{P}$ & 5'-FAM- TCCTGATCCAGTTCCTGCAGAAAAAGGCA-TAMRA-3' \\
\hline \multirow[t]{2}{*}{ TNF- $\alpha$ (NM_000594) } & $\mathrm{F}$ & 5'- CCACCACGСTCTTCTGCC-3' \\
\hline & $\mathrm{P}$ & 5'-FAM- CACTTTGGAGTGATCGGCCCC-TAMRA-3' \\
\hline \multirow[t]{3}{*}{ MUC5B (NM_012234) } & $\mathrm{F}$ & 5'- GAAGCTGCGACAGGCAAG-3' \\
\hline & $\mathrm{R}$ & 5'- CCTCAGCCCTGAGTTACCC-3' \\
\hline & $P$ & UPL\#40 (Universal Probe Library, Roche) \\
\hline GAPDH (NM_002046) & $P$ & 5'-FAM-CGTCCCCAGCCGAGCCACATCG -TAMRA-3' \\
\hline
\end{tabular}

F: forward primer, R: reverse primer, P: TaqMan probe. IL-6, Interleukin-6; TNF- $\alpha$, tumor necrosis factor-alpha; GAPDH, glyceraldehyde-3phosphate dehydrogenase. 